\title{
Spectroscopic signatures of topological and diatom-vacancy defects in single-walled carbon nanotubes
}

\author{
Wissam A. Saidi and Patrick Norman
}

\section{Linköping University Post Print}

\section{Tweet}

N.B.: When citing this work, cite the original article.

Original Publication:

Wissam A. Saidi and Patrick Norman, Spectroscopic signatures of topological and diatomvacancy defects in single-walled carbon nanotubes, 2014, Physical Chemistry, Chemical Physics - PCCP, (16), 4, 1479-1486.

http://dx.doi.org/10.1039/c3cp53762e

Copyright: Royal Society of Chemistry http://www.rsc.org/

Postprint available at: Linköping University Electronic Press http://urn.kb.se/resolve?urn=urn:nbn:se:liu:diva-103714 
Cite this: Phys. Chem. Chem. Phys., 2014, 16, 1479

Received 4th September 2013, Accepted 21st October 2013

DOI: $10.1039 / c 3 c p 53762 e$

www.rsc.org/pccp

\section{Spectroscopic signatures of topological and diatom-vacancy defects in single-walled carbon nanotubes}

\begin{abstract}
Wissam A. Saidi*a and Patrick Norman ${ }^{b}$
The optical properties, including UV-vis spectra and resonance Raman profiles, of pristine and defected single-walled carbon nanotubes (SWCNTs) are computed using state-of-the-art time-dependent density functional theory (TDDFT) as implemented using the Liouville-Lanczos approach to linear-response TDDFT. The CNT defects were of the form of Stone-Wales and diatom-vacancies. Our results are in very good agreement with experimental results where defects were introduced into a part of defect-free CNTs. In particular, we show that the first and second $\pi-\pi^{\star}$ excitation energies are barely shifted due to the defects and associated with a relatively small reduction in the maxima of the absorption bands. In contrast, the resonance Raman spectra show close to an order of magnitude reduction in intensities, offering a means to distinguish between pristine and defected SWCNTs even at low defect concentrations.
\end{abstract}

\section{Introduction}

Carbon nanotubes (CNTs) in their pristine or functionalized forms have been extensively studied due to their remarkable electronic and structural properties, ${ }^{1-4}$ and are widely used in different applications including biomedical sensing, electronics, and charge transport. ${ }^{5,6}$ According to tight-binding models, the electronic nature of CNTs is determined by their chirality index $(n, m)$, which defines the folding vector along which a graphite sheet is rolled up in order to form CNTs. The $(n, n)$ armchair CNTs are metallic whereas, if $(n-m) \bmod 3=0$, the $(n, 0)$ zigzag and $(n, m)$ chiral tubes are metallic or quasimetallic with a very narrow band gap, and semiconducting for $(n-m) \bmod 3 \neq 0 .{ }^{5,6}$

In practice, however, this clear and simple characterization is affected by the many defects that can occur on CNTs, including topological Stone-Wales (SW), mono-vacancies, and diatom-vacancies (DVs). Defects are known to affect greatly the physicochemical properties of the CNTs. For example, SW defects are chemically very active ${ }^{7}$ and their presence abruptly changes the mechanical and electronic properties of CNTs such as e.g. closing the gap in large-gap CNTs or opening the gap in small-gap nanotubes. ${ }^{8}$ The sensitivity of SWCNTs can be significantly enhanced by introducing a low density of defects along the nanotube sidewall as defect sites form low-energy

\footnotetext{
${ }^{a}$ Department of Chemical and Petroleum Engineering, University of Pittsburgh, Pittsburgh, PA, 15261, USA. E-mail: alsaidi@pitt.edu

${ }^{b}$ Department of Physics, Chemistry and Biology, Linköping University,

SE-581 83 Linköping, Sweden
}

adsorption sites that can dominate the electrical responses of the CNTs to the presence of chemical vapors. ${ }^{9}$ Therefore, it is of prime importance to study the structural and physical properties of defected CNTs, and provide a means to distinguish between the different defect types.

Carbon nanotube characterization relies extensively on Raman spectroscopy, ${ }^{10}$ and it is very appealing to apply optical methods to distinguish between defect types of CNTs. The $\mathrm{D}$-band in the Raman spectra of $\mathrm{sp}^{2}$ carbons is a signature of defects in the graphene lattice ${ }^{11}$ and is often used to characterize defective CNTs. However, the interpretation of the D-band feature might be ambiguous as a large observed D-band intensity might originate from one nanotube while the radial breathing mode (RBM) or graphitic G-band could be due to another tube present in the same sample. Recently, the influence of the defects on the Raman features was explored by Kalback and co-workers where spectroscopic signatures of defected CNTs were investigated. ${ }^{12}$ In this study, a low concentration of defects was created intentionally on a part of an originally defect-free individual semi-conducting SWCNT, and, in these experiments, doping was shown to lead to a broadening and a decrease in the maximum intensity of the resonance profile. ${ }^{12}$ It was also demonstrated that the maxima of the resonance profiles for the defected and pristine CNTs do not coincide; the pristine and defected CNTs were most effectively excited by laser pulses of energies 2.05 and $2.10 \mathrm{eV}$, respectively. ${ }^{12}$

However, despite the extensive use of RR spectroscopy techniques for investigating CNTs experimentally, CNT characterization is still challenging mostly because of the lack of analytically pure bulk CNT samples. Also, the interpretation of 
the Raman resonance profile is challenging as well because of its sensitivity to the local environment. ${ }^{13}$ Theoretical modeling of the resonance profile, especially using first-principles methods, can address adequately both of these challenges. However, the computation of RR scattering cross sections is still far from being routine calculations, especially for extended systems. Popov and co-workers have investigated RR profiles for pristine and defected CNTs ${ }^{14,15}$ using a tight binding approach, but this method is of limited use due to its poor predictive power and also the difficulties in extending it to functionalized CNTs. For example, a standard tight-binding approach predicts equally spaced valence and conduction bands, ${ }^{5,16}$ which is only true for large diameter CNTs. In the case of small-diameter CNTs, the curvature-induced hybridization between $\sigma-\pi$ bands makes the van Hove singularities asymmetric with respect to the Fermi energy. ${ }^{17}$

In this paper, we study the spectroscopic properties of pristine and defected $(7,0)$ CNTs, where defects are of the form of SW and DV defects. The mono-vacancies are not investigated due to their high formation energies. The $(7,0)$ CNT is the smallest non-metallic zigzag CNT which was selected due to computational demands. The $(5,0)$ CNT has a smaller diameter and it is insulating according to the band-folding scheme but found to be metallic due to strong curvature effects. ${ }^{18}$ Nevertheless, our findings are expected to apply to larger CNTs as well. Defects will lower the symmetry of the CNT resulting in an increase in the number of Raman-active modes, but here we select the RBM and the G-band that are the dominant Raman modes in CNTs. Our results reproduce the recent experimental results, ${ }^{12}$ namely that the Raman shifts and intensities are sensitive to the CNT defects, while the UV-vis spectra show little shifts in the peaks. In addition, we show that low energy peaks in the UV-vis spectra are induced by the defects. But these peaks have relatively very small intensities, which explain why they have not been observed experimentally.

The rest of the paper is organized as follows. First, we give a brief review of the approach that we used to compute the RR cross sections to be followed by a detailed presentation of the CNT models and the complexities associated with a cluster approach. We then show and discuss our results and conclude with a brief summary and outlook.

\section{Methodology}

The Raman scattering cross section is determined from the transition polarizability tensor. Each vibrational normal mode $\nu$ with angular frequency $\omega_{\nu}$ gives rise to a dipole oscillating with angular frequency $\omega-\omega_{\nu}$ and with an intensity proportional to the differential cross section: ${ }^{19}$

$$
\frac{\mathrm{d} \sigma_{\nu}}{\mathrm{d} \Omega}=\frac{1}{16 \pi^{2} \varepsilon_{0}^{2} c^{4}} \frac{\left(\omega-\omega_{\nu}\right)^{4}}{[1-\exp ]\left(-\hbar \omega_{\nu} / k_{\mathrm{B}} T\right)} \times S_{\nu},
$$

where $T$ is the temperature and $S_{\nu}$ is the scattering factor, or the Raman activity, which is determined from the transition polarizability tensor by an expression that depends on the experimental setup. ${ }^{20,21}$ Assuming that the detection is made over all scattered polarizations along the $X$-direction and perpendicular to the $Z$-direction of propagation as well as the $Y$-axis along which the incident light of frequency $\omega$ is polarized, the relevant expression for the scattering factor becomes equal to ${ }^{20}$

$$
S_{\nu}=\left|\overline{\alpha_{Y Y}^{\mathrm{f} 0} \alpha_{Y Y}^{\mathrm{f} 0}}+\overline{\alpha_{Z Y}^{\mathrm{f} 0} \alpha_{Z Y}^{\mathrm{f} 0}}\right| .
$$

Here $\alpha_{\alpha \beta}^{\mathrm{fo}}$ is the $\alpha \beta$ term of the transition polarizability between the initial (ground) $\left|\nu_{0}\right\rangle$, and final $\left|\nu_{\mathrm{f}}\right\rangle$ vibrational states both belonging to the electronic ground state. For a randomly oriented sample, the two terms on the right-hand side of eqn (2) are expressed in the molecular frame in terms of the isotropic, $\overline{\alpha^{\mathrm{f} 0}}$, and anisotropic averages, $\gamma$, of the transition polarizability tensor such that,

$$
\begin{gathered}
\overline{\alpha_{Y Y}^{\mathrm{f} 0} \alpha_{Y Y}^{\mathrm{f} 0}}=\overline{\alpha^{\mathrm{f} 0}}+\frac{4}{45} \gamma^{2}, \\
\overline{\alpha_{Z Y}^{\mathrm{f} 0} \alpha_{Z Y}^{\mathrm{f} 0}}=\frac{1}{15} \gamma^{2}, \\
\overline{\alpha^{\mathrm{f} 0}}=\frac{1}{3}\left(\alpha_{x x}^{\mathrm{f} 0}+\alpha_{y y}^{\mathrm{f} 0}+\alpha_{z z}^{\mathrm{f} 0}\right), \\
\gamma^{2}=\frac{1}{2}\left[\left(\alpha_{x x}^{\mathrm{f} 0}-\alpha_{y y}^{\mathrm{f} 0}\right)^{2}+\left(\alpha_{x x}^{\mathrm{f} 0}-\alpha_{z z}^{\mathrm{f} 0}\right)^{2}+\left(\alpha_{y y}^{\mathrm{f} 0}-\alpha_{z z}^{\mathrm{f} 0}\right)^{2}\right. \\
\left.+6\left(\alpha_{x y}^{\mathrm{f} 0} \alpha_{x y}^{\mathrm{f} 0}+\alpha_{x z}^{\mathrm{f} 0} \alpha_{x z}^{\mathrm{f} 0}+\alpha_{y z}^{\mathrm{f} 0} \alpha_{y z}^{\mathrm{f} 0}\right)\right] .
\end{gathered}
$$

In the short time approximation, $\alpha_{\alpha \beta}^{\mathrm{fo}}$ is determined in the harmonic approximation as

$$
\alpha_{\alpha \beta}^{\mathrm{f} 0}=\sqrt{\frac{\hbar}{2 \omega_{\nu}}}\left(\frac{\partial \alpha_{\alpha \beta}^{\mathrm{e}}}{\partial Q_{\nu}}\right)_{\mathrm{eq}},
$$

where $\alpha_{\alpha \beta}^{\mathrm{e}}$ is the (complex) electronic polarizability, and $Q_{\nu}$ is the normal mode coordinates of the $\nu$ th mode. The derivative of the electronic polarizability with respect to $Q, \frac{\partial \alpha_{\alpha \beta}^{\mathrm{e}}}{\partial Q_{\nu}}$, is evaluated for the equilibrium geometry, and is the key quantity that needs to be determined for the evaluation of eqn (7). If the optical frequency is in near resonance, or resonance, with electronic transitions in the molecular system, then the polarizability calculations must be based on a resonance convergent response theory. Inclusion of relaxation in wave function theory was made by Norman and co-workers ${ }^{22,23}$ by means of a formulation of wave mechanics based on the Ehrenfest theorem, and the formulation, also referred to as complex polarization propagator (CPP) theory. ${ }^{22-26}$

In our calculations, the electronic polarizability is computed for the periodic CNT with TDDFT as implemented using density-functional perturbation theory (DFPT). TDDFT is a very good compromise between speed and accuracy that can be applied to relatively large systems as compared to more accurate approaches based on equation of motion coupled cluster or Bethe-Salpeter methods. This is particularly true for TDDFPT that can handle relatively large systems as compared 
to traditional approaches. The TDDFPT Lanczos approach has been introduced recently, and its main advantage is that it allows for the calculations of the entire absorption spectrum (and hence also the polarizability) of the system at a cost comparable to that of the ground state electronic structure optimization. $^{27}$ Contrary to conventional approaches, this method avoids the resolution of individual states, and is thus well suited for large systems with large basis sets. The Lanczos technique has also been adopted in vibrational structure theory due to the high density of states ${ }^{28}$ and in damped coupled cluster electronic response theory. ${ }^{26}$

Briefly, in TDDFPT the electron dynamics is described using TD Kohn-Sham (KS) equations for the occupied (valence) orbitals as,

$$
i \frac{\partial \phi_{v}(\mathbf{r}, t)}{\partial t}=\mathscr{H}_{\mathrm{KS}}(t) \phi_{v}(\mathbf{r}, t),
$$

where $\mathscr{H}_{\mathrm{KS}}$ is the KS Hamiltonian defined as,

$$
\mathscr{H}_{\mathrm{KS}}(t)=-\frac{1}{2} \nabla^{2}+v_{\mathrm{ext}}(\mathbf{r}, t)+v_{\mathrm{HXC}}(\mathbf{r}, t) .
$$

Here $\nu_{\text {ext }}(\mathbf{r}, t)$ is the external potential, and $\nu_{\mathrm{Hxc}}(\mathbf{r}, t)$ is the Hartree-exchange-correlation (HXC) potential. Linearization of the TD KS equation with respect to external potential implies

$$
i \frac{\mathrm{d} \rho^{\prime}(t)}{\mathrm{d} t}=\left[H_{\mathrm{KS}}^{0}, \rho^{\prime}(t)\right]+\left[V_{\mathrm{HXC}}^{0}, \rho^{0}\right]+\left[V_{\mathrm{ext}}{ }^{\prime}(t), \rho^{0}\right],
$$

where $\rho^{0}$ is the unperturbed electron density, $\rho^{\prime}(t)=\rho(t)-\rho^{0}$ is the response charge density defined as the deviation of the instantaneous electron density $\rho(t)$ from the unperturbed density $\rho^{0}, V_{\text {ext }}{ }^{\prime}(t)$ is the external perturbation, and $V_{\mathrm{HXC}}{ }^{\prime}$ is the response HXC potential. Eqn (10) can be recast into:

$$
(\omega-\mathscr{L}) \cdot \rho^{\prime}(\omega)=\left[V_{\text {ext }}{ }^{\prime}(\omega), \rho^{0}\right],
$$

where $\mathscr{L}$ is the Liouvillian super-operator whose action onto $\rho^{\prime}$ is defined as:

$$
\mathscr{L} \cdot \rho^{\prime}=\left[H_{\mathrm{KS}}^{0}, \rho^{\prime}\right]+\left[V_{\mathrm{HXC}}\left[\rho^{\prime}\right], \rho^{0}\right] .
$$

The electronic polarizability, $\alpha_{\alpha \beta}^{\mathrm{e}}(\omega)$, at any frequency $\omega$ can then be expressed as an off-diagonal element of the resolvent of $\mathscr{L}$. The inversion of $\mathscr{L}$ is avoided by using an iterative Lanczos recursion scheme, ${ }^{27}$ which is necessary when the TDDFPT is employed in combination with a large basis set such as plane waves.

\section{Computational details}

All ground state results are obtained by first principles calculations in the framework of DFT, using the PWSCF package of the Quantum Espresso distribution. ${ }^{29}$ In this scheme, core electrons are replaced by ultrasoft pseudopotentials. The KS reference state and the valence electron densities are expanded using plane waves with a cutoff of 20 and 160 Ry, respectively. The spin-averaged generalized-gradient approximation (GGA) by Perdew, Burke, and Ernzerhof $(\mathrm{PBE})^{30}$ is employed throughout, and we restricted the sampling of the Brillouin zone (BZ) to the $\Gamma$-point only. Equilibrium geometries are obtained by relaxing all atomic coordinates using a convergence threshold of $10^{-4}$ Ry per Bohr on the atomic forces, and $10^{-7}$ Ry on the energies of the self-consistent step. The use of periodic boundary conditions imposes a relatively high concentration of defects, which is an artifact that cannot be rectified without unrealistic increases in computational costs. To study the effects of the defect concentration on the results, we used a supercell approach with three and four repeat unit cells along the tube axis where the lattice constant is fixed at $4.32 \AA$. In each case, the optimum ground state configuration is found for the pristine and defected CNTs. For the UV-vis spectrum, we also examined a six repeat unit CNT. The images along the non-periodic directions in the supercell are isolated from each other by more than $10 \AA$ to minimize fictitious interactions between them.

The phonon spectra are computed using a finite displacement approach utilizing the Phonopy program ${ }^{31}$ with a displacement of $0.01 \AA$ along $\pm x, \pm y$, and $\pm z$ directions from the equilibrium position. The self-consistent step was converged using a tight convergence of $10^{-8}$ Ry on the energies in order to ensure sufficient numerical accuracy in the calculation of forces.

Optical properties, including the absorption spectrum and the Raman intensities, are computed using TDDFT as implemented in DFPT in conjunction with the PBE XC functional. Local and semi-local functionals are known to underestimate excitation energies, and hybrid functionals would make a better alternative, but we were forced to restrain from this choice due to the steep computational cost involved with the use of hybrid functionals in combination with periodic calculations and plane-wave basis sets. To obtain well converged spectra, we have used between 2000 and 3000 Lanczos iterations for the pristine CNTs and between 5000 and 8000 iterations for the defected ones. The larger number of iterations for defected CNTs compared to the pristine ones is due to their loss of symmetry. The polarizabilities are computed using an extrapolation procedure, where up to 10000 iterations have been adopted. $^{27}$

The absorption spectra are obtained from the imaginary value of the polarizability where a damping parameter is used to mimic various broadening effects occurring under experimental conditions, a technique that was developed by Norman and co-workers. ${ }^{22,23,32}$ The value of the damping parameter $\Gamma$ (HWHM) does not determine only the line profile in the absorption spectrum but also the validity of the approximations that are made to compute the Raman spectra. In our previous study, we found that a reasonable choice for $\Gamma$ for describing the $\pi \pi^{*}$-resonance enhanced transition polarizability for the $\mathrm{C}-\mathrm{C}$ stretch mode in the ethylene model system is around $200 \mathrm{meV} .{ }^{33}$ In the present study, we will adapt the same value knowing that our predicted absolute cross sections may be somewhat reduced compared to experimental results. ${ }^{33}$ However, it is noteworthy that it is computationally trivial to adapt a different value of $\Gamma$ in the TDDFPT approach, and for illustration purposes we also compute the UV-vis spectra using two additional values of $\Gamma$. 
The derivatives of the polarizabilities of eqn (7) are carried out using a two point numerical differentiation scheme. This is done by first performing a normal mode analysis of the ground state equilibrium structure, and then obtaining the massweighted normal coordinates $Q$ by multiplying the eigenmodes by $1 / \sqrt{M}$ where $M$ is the mass matrix. The finite displacements are then generated along $Q$ with step lengths of $\pm 0.01 \times \sqrt{\mathrm{amu}}$ where $\mathrm{amu}=1822.8884843$.

Mostly because one can employ different hybrid density functionals with a maintained reasonable computational cost, it would have been advantageous to carry out the present study by using a finite cluster approach and by capping the CNT segments with hydrogen atoms. We have demonstrated previously that a cluster approach can provide a fair description of the structure, absorption spectra, and RR profiles of $(7,0)$ CNTs that are decorated with $\mathrm{H}$ and $\mathrm{F}^{33}$ However, for the defected CNTs we found that the cluster approach, even with up to 10 unit cells of the $(7,0) \mathrm{CNT}$, had substantial size effects when defects are introduced on the CNTs. These effects caused a significant bending of the CNT near the tube defect site and which must be considered to be an artifact of the cluster approach as we have not seen it in the periodic calculations.

\section{Results and discussion}

\subsection{Equilibrium geometries and vibrational frequencies}

We investigated defects that involve a pair of bonded carbon atoms, namely SW and DV defects. For each type, there are two kinds of bonded carbon atoms resulting in two isomers. Here, we selected the isomer where the two bonded carbon atoms are along the tube axis which have the lowest formation energy. The equilibrium structures of pristine and defected CNTs with four units are illustrated in Fig. 1. The SW defect is the result of a rotation by $\pi / 2$ of two carbon atoms about their bond center, which turns the four neighboring hexagons after reconstruction into two heptagons as shown in Fig. 1(b). On the other hand, after the removal of the two bonded carbon atoms, the reconstruction of the CNT with a DV as shown in Fig. 1(c) results in a stable pentagon-octagon-pentagon (or 5-8-5) arrangement, in agreement with previous studies. ${ }^{34,35}$

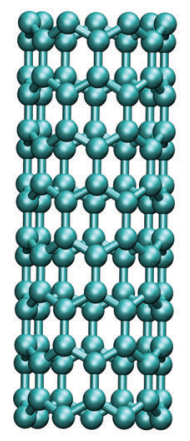

(a)

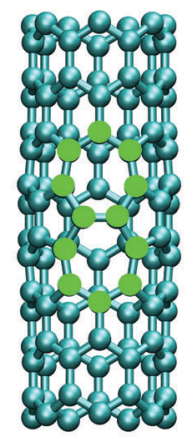

(b)

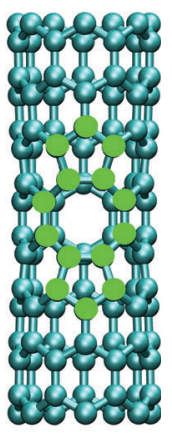

(c)
Fig. 1 Structures of single-walled $(7,0)$ CNTs: (a) pristine CNT, (b) CNT with Stone-Wales defects and (c) CNT with double vacancy defects. Atoms involved in the SW and DV defects are highlighted in light-green.
Table 1 Vibrational frequencies (in $\mathrm{cm}^{-1}$ ) for the RBM and G-band of pristine and SW- and DV-defected $(7,0)$ CNTs

\begin{tabular}{llllll}
\hline & 3 units & & & \multicolumn{2}{l}{4 units } \\
\cline { 2 - 3 } & RBM & G & & RBM & G \\
\hline P & 401.3 & 1607.2 & 404.5 & 1567.6 \\
SW & 400.2 & 1568.5 & 399.0 & 1554.3 \\
DV & 403.6 & 1577.4 & 405.5 & 1558.7
\end{tabular}

Table 1 summarizes the vibrational frequencies of the RBM and G-band. The RBM is a low frequency mode characterized by an in-phase radial motion of the carbon atoms with a mode frequency that is inversely proportional to the CNT diameter. For the pristine CNT, the RBM is located at 401.3 and $404.5 \mathrm{~cm}^{-1}$ for the three- and four-unit CNTs, respectively. These results are in good agreement with the previous results of $418.5 \mathrm{~cm}^{-1}$ obtained with a local atomic orbital basis set of double- $\zeta$ quality. ${ }^{36}$ Upon CNT defection, the frequency of the RBM barely changes-as compared to the pristine CNT, the SW and DV defected systems show minor red- and blue-shifts, respectively, which agrees with tight binding calculations on $(7,0) \mathrm{CNT}^{15}$ It should be emphasized that the character of the RBM modes for the defected CNTs is associated with an in-phase radial motion of the carbon atoms but in contrast to the pristine CNT the vibrations are not the same in magnitude and few atoms are either at rest or have out-of-phase motions with respect to most of the other atoms.

The G-band is characterized by the out-of-phase vibrations of the neighboring carbon atoms parallel to the tube surface. Being associated with planar carbon vibrations, the G-band has a higher vibrational frequency, in the region of values for aromatic $\mathrm{C}-\mathrm{C}$ ring-stretch motions. For the pristine CNTs, the G-band is located at 1607 and $1567 \mathrm{~cm}^{-1}$ for the three- and four-unit systems, respectively, and, for both defect types, this mode is red-shifted by some $10-30 \mathrm{~cm}^{-1}$. The sign of the shift in our calculations is found to be opposite to results obtained with a tight-binding approach, where the G-band of SW and DV defected CNTs was shown to blue-shift by $23-60 \mathrm{~cm}^{-1} \cdot{ }^{15}$

It is interesting to note that for both the RBM and the G-band, the frequencies of the CNTs with a DV defect are larger than the corresponding values for the CNTs with the SW defect. However, as can be seen from the table, the difference is too small to be resolved experimentally. Additionally, overall the results and trends are consistent between systems with three and four unit cells, namely that for both defect types, the RBM barely changes due to defects while the G-band is more significantly altered. The results clearly show that the shift is proportional to the density of the defects for the G-band whereas the RBM is less sensitive. It is expected that the Raman shifts of both modes will not show any sensitivity to the defects in the low concentration limit.

\subsection{UV-vis absorption spectrum}

In Fig. 2, we present the UV-vis absorption spectra for the pristine and defected CNTs in the range of $0-7 \mathrm{eV}$ with three, four, and six repeat units. Due to the high computational 


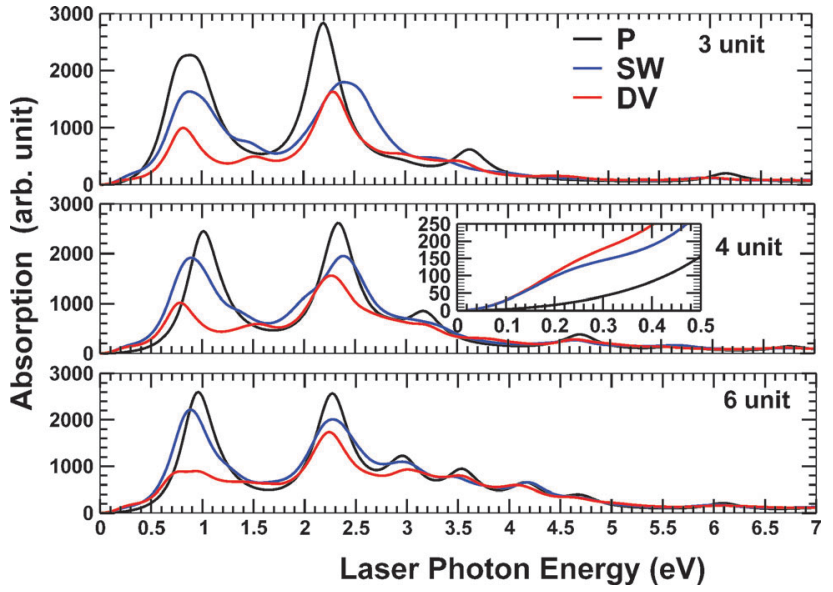

Fig. 2 UV-vis absorption spectra for pristine and defected $(7,0)$ CNTs with 3,4 and 6 repeat units. The inset in the middle panel shows the absorption spectrum between 0 and $0.5 \mathrm{eV}$ to emphasize that defects induce a low absorption band that is absent in the pristine CNTs.

demands, we restrict our study to encompass the $\pi \pi^{*}$-transitions that, in the electric-dipole approximation, couple to the ground state by means of an electric field polarized parallel to the tube axis. However, this is nevertheless a very good approximation especially in the extended system because the excitations that are perpendicular to the tube axis have a smaller oscillator strength due to the depolarizing field that originates from bound surface charges. ${ }^{37,38}$ This was validated in our recent study using a cluster approach. ${ }^{33}$

The UV-vis spectra shown in Fig. 2 for the pristine CNTs display mainly the peaks corresponding to the $S_{11}$ and $S_{22}$ excitations. Table 2 summarizes the excitation energies and the band maxima. We avoided the computation of the oscillator strengths of the excitations because there is no simple way to do so from the spectrum, as every near-lying state will contribute to the peak maximum and not all excitations energies are resolved using the $\Gamma=200 \mathrm{meV}$ value. As can be seen from the table, the first and second optical excitation energies are located at 0.96 and $2.27 \mathrm{eV}$ for the six unit CNT. For comparison, we note that the experimental values for the first and second optical excitation energies are 1.29 and $3.14 \mathrm{eV},{ }^{39}$ respectively, and the corresponding theoretical benchmarks, obtained using a Bethe-Salpeter approach on the extended system, are equal to 1.20 and $3.00 \mathrm{eV}^{40}$ The underestimation of excitation energies that we observe in our results is to be expected and is caused by our use of a semi-local exchangecorrelation functional.

Table 2 Excited state energies (eV) and maxima of the corresponding absorption bands (arbitrary units) for the $S_{11}$ and $S_{22}$ excitations

\begin{tabular}{|c|c|c|c|c|c|c|}
\hline & \multicolumn{2}{|l|}{3 unit } & \multicolumn{2}{|l|}{4 unit } & \multicolumn{2}{|l|}{6 unit } \\
\hline & $S_{11}$ & $S_{22}$ & $S_{11}$ & $S_{22}$ & $S_{11}$ & $S_{22}$ \\
\hline$P$ & $0.89(227)$ & $2.19(284)$ & $1.01(245)$ & $2.32(261)$ & $0.96(260)$ & $2.27(257)$ \\
\hline SW & $0.88(163)$ & $2.40(180)$ & $0.89(192)$ & $2.38(195)$ & $0.88(222)$ & $2.27(201)$ \\
\hline DV & $0.81(100)$ & $2.29(163)$ & $0.77(102)$ & $2.27(156)$ & $0.85(88)^{*}$ & $2.23(174)$ \\
\hline
\end{tabular}

* This appears as a double peak at 0.8 and $0.94 \mathrm{eV}$ with equal intensity.
The effects of the defects on the UV-vis spectra are not substantial. First, as can be seen from Table 2 and Fig. 2, the $S_{11}$ and $S_{22}$ excitation energies show a relatively small shift. For example, for the pristine, and SW and DV defected CNTs with six units, the $S_{11}$ excitations energies are located at $0.96,0.88$ and $0.85 \mathrm{eV}$, while the $S_{22}$ excitations are located at 2.27, 2.27 and $2.23 \mathrm{eV}$, respectively. In comparison with the excitations energies, the band maxima of these excitations show a decrease, which is inline with the reduced RR intensities as we will discuss later.

The small shift of the excitation energies due to defects agrees with previous results on defected CNTs. Kilina et $_{\text {al. }}{ }^{41}$ showed that hydrogen defects caused a very small blue-shift in the $\pi-\pi^{*}$ excitation energy. We also found that the $S_{11}$ excitation of hydrogen and fluorine decorated CNTs are blue-shifted by less than $0.1 \mathrm{eV}$ compared to the pristine CNT. ${ }^{33}$ Furthermore, the reduction of the band maxima for the defected CNTs that is seen here is also in line with our previous results, where the oscillator strength of the first optical excitation of hydrogen and fluorine decorated CNTs is reduced by some $30-50 \%$.

Another effect of the SW and DV defects on the UV-vis spectra is that new weak absorption bands are induced that did not exist in the pristine CNT spectrum. This can be seen from the inset of the middle panel of Fig. 2, which clearly shows that the two defected CNTs have a small peak around $0.25 \mathrm{eV}$ that is absent in the spectra of the pristine CNT. It should be warranted that the small value of this low excitation energy could be due to the underestimation of the excitation energies in semi-local DFT functionals. For comparison, we note that in $\mathrm{H}$ - and F-functionalized CNTs, a low energy induced peak was also observed in the spectra upon functionalization. ${ }^{33,41}$ For example, for the F-functionalized CNTs a low energy peak at $\approx 1 \mathrm{eV}$ was reported before and with an oscillator strength that is only a factor of $2-3$ smaller than that of the $\pi-\pi^{*} S_{11}$ excitation. For this low energy excitation, the orbitals involved in the transition clearly showed that both the adatoms and the CNTs are involved in the excitation. ${ }^{33}$

In the TDDFPT approach, the polarizability is computed directly and thus not every excitation can be resolved in the UV-vis spectra. Peaks will be hidden or obscured if they have very small oscillator strengths or if they appear close to dominant excitations. This situation is different from conventional spectral calculations in which case all excited states are individually resolved regardless of their absorption strengths. In general, additional peaks that are close to the main excitation require a smaller value of the imaginary value of $\Gamma$ to be resolved. To show that this is indeed the case, we show in Fig. 3 the UV-vis spectra as obtained using two different values of $\Gamma=400$ and $100 \mathrm{meV}$ for the 6 unit CNT. The spectra using $\Gamma=200$ are shown in Fig. 2. As can be seen from Fig. 2 and 3, the spectra obtained using $\Gamma=400 \mathrm{meV}$ are fairly similar to those using $\Gamma=200 \mathrm{meV}$ near the $S_{11}$ and $S_{22}$ bands. On the other hand with $\Gamma=100 \mathrm{meV}$, the DV first main absorption band $S_{11}$ that is seen with $\Gamma=200 \mathrm{meV}$ corresponds to two absorption bands, and for the SW defect the second peak corresponds to two excitations as well. For comparison, the 


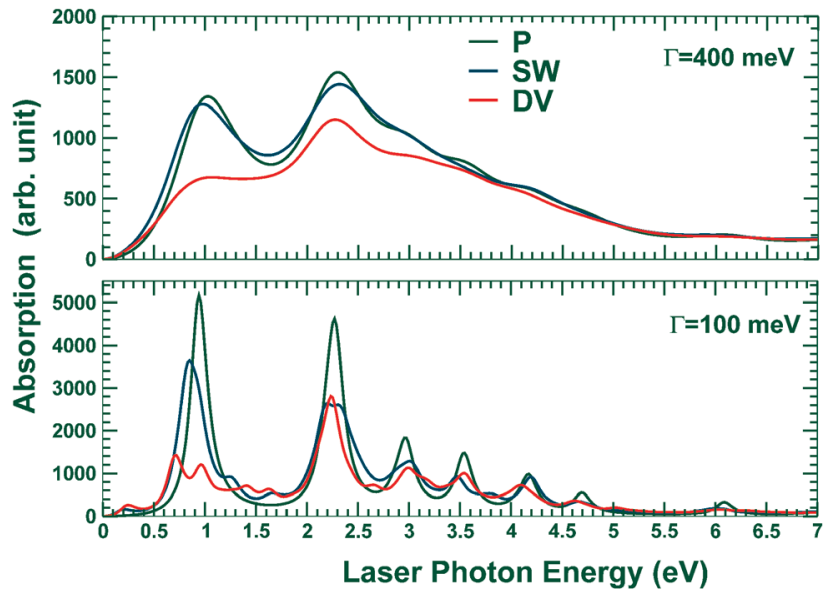

Fig. 3 UV-vis absorption spectra for pristine and defected $(7,0)$ CNTs with 6 repeat units as obtained using two different values of $\Gamma$. The spectra with $\Gamma=200$ meV are shown in Fig. 2.

pristine CNT shows only two main bands $S_{11}$ and $S_{22}$, regardless of the value of $\Gamma$. The presence of the additional peaks in the defected CNTs compared to the pristine one was reported before in a tight-binding study of the RR profile of a $(7,0) \mathrm{CNT}^{15}$

The excitation energies for the pristine and defected CNTs show little dependence on the number of units of the CNT. The band maxima show a systematic small increase with system size, an indication of the delocalized nature of the exciton. In these calculations, it is clear that the UV-vis spectra are not very sensitive to the size of the CNT. This is very encouraging considering that the optical properties as computed with a cluster approach, where the dangling bonds are saturated with hydrogen atoms, showed significant dependence on the number of units that are used to model the CNT. For instance, in our previous study of the $(7,0)$ CNT using a cluster approach with functional CAM-B3LYP, we found that the first optical excitation for the $(7,0) \mathrm{CNT}$ is located at $3.06 \mathrm{eV}$ for the 4 unit cluster, and at $2.51,2.26,2.08 \mathrm{eV}$ for the 6,8 , and 10 unit clusters, respectively. ${ }^{33}$ The corresponding oscillator strengths of these excitations increase with the tube length from 1.20 for 4 units to 7.38 for 10 units.

Finally, we note that the spectra of the pristine and defected CNTs are fairly similar which make it challenging to distinguish the two defected CNTs, or even to distinguish the pristine from the defected CNTs based solely on UV-vis absorption spectroscopy. With $\mathrm{H}-$ and F-functionalization, the pristine and defected CNTs can potentially be distinguished based on the low-energy bright exciton which exist only in the defected CNTs. ${ }^{33}$ In contrast, the low energy band that is observed for the defected CNT has a very low intensity, which is unlikely to be detected. Additionally, the extra peaks that can be seen in the spectra of Fig. 3 using $\Gamma=100 \mathrm{meV}$ cannot be resolved experimentally. ${ }^{41}$ For defected CNTs, however, we will show next that the RR spectra are more sensitive to the defect chemistry.

\subsection{RR enhancement of the RBM and G-band}

We determine the resonant Raman cross sections for the RBM and the G modes, which are the most symmetry-allowed Raman

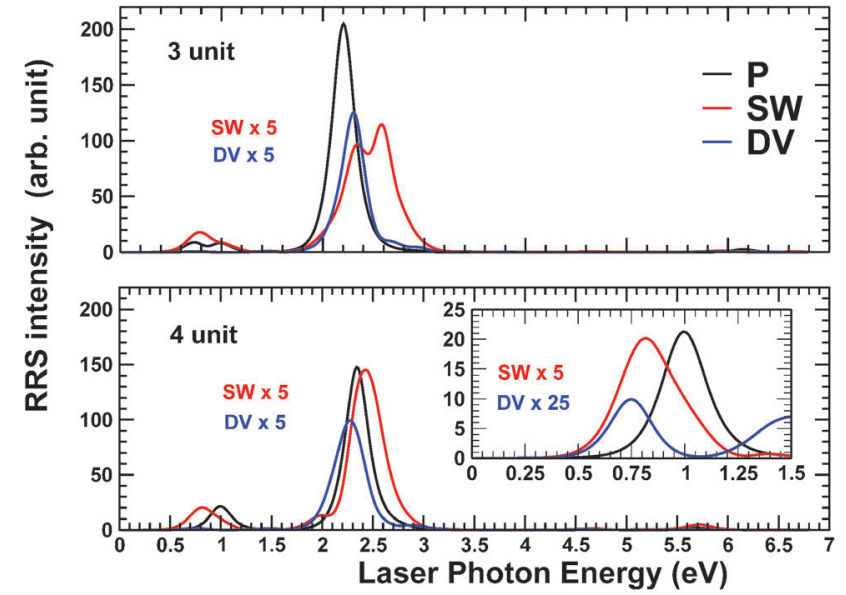

Fig. 4 RBM Resonance Raman profile for pristine and defected $(7,0)$ CNTs with 3 and 4 repeat units. The inset for the 4 repeat unit CNTs shows the $\mathrm{RR}$ intensities for photon energies around the first absorption band.

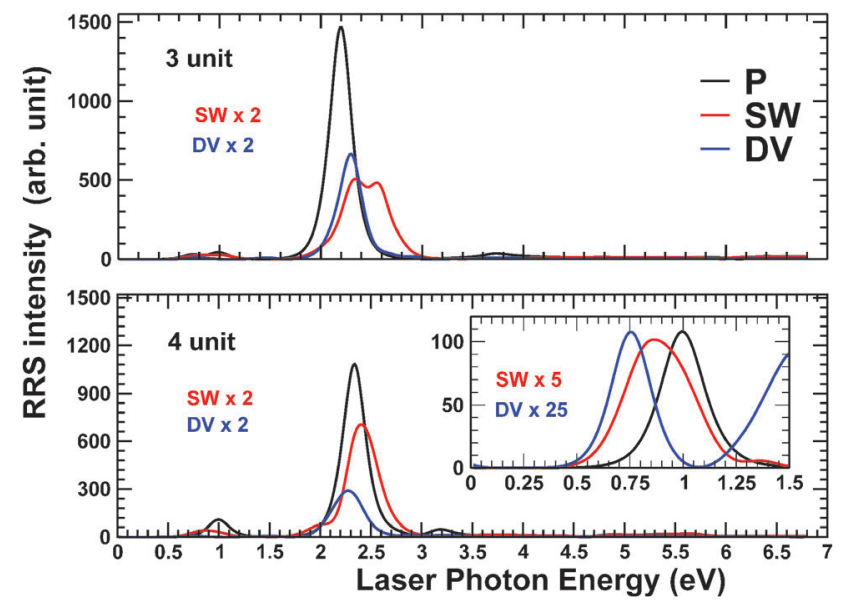

Fig. 5 The same as Fig. 4 but for the G-band.

active modes. The dependency of the scattering cross section on the laser energy is shown in Fig. 4 for the RBM and in Fig. 5 for the G-band; the 3 and 4 unit cell sizes are considered in these calculations. The RR spectra show peaks around about 1 and $2.3 \mathrm{eV}$, which correspond to the $S_{11}$ and $S_{22} \pi-\pi^{*}$ excitations, respectively, and which were discussed above, in concern with the UV-vis spectra.

In contrast to the small shifts in the laser frequencies between spectral peaks of pristine and defected CNTs, Fig. 4 and 5 show that defects induce an almost one order of magnitude reduction in the $\mathrm{RR}$ intensities. The reduction of the intensities can be anticipated by comparing the corresponding atomic vibrations of the modes for the pristine and defected CNTs. For example, both SW and DV defects cause some of the carbon atoms that are close to the defect to have a small, or even a vanishing, amplitude of vibration. Thus it is expected that the orbital densities that are close to these atoms contribute less to the derivative of the electronic polarizability of eqn (7) when perturbed along the corresponding vibrational mode. This explains the reduction of the intensity for the CNTs 
with defects. Similar effects were also observed before in our study of $\mathrm{H}$ - and F-functionalized CNTs. ${ }^{33}$ Also, the reduction of Raman intensities was observed in CNTs after acid treatment with $\mathrm{H}_{2} \mathrm{SO}_{4} / \mathrm{HNO}_{3}{ }^{13}$ This is also the case after functionalization by $\mathrm{CCl}_{2}, \mathrm{COOH}$, and $\mathrm{COCCl}_{3}{ }^{42}$ The general qualitative agreement in the RR spectra found between CNTs with SW or DV defects and those of functionalized CNTs stems from the fact that in all of these cases the defect disrupts locally the CNT $\mathrm{sp}^{2}$ network in a similar manner.

Additionally, as can be seen from the RR profiles in Fig. 4 and 5, the intensity of the CNT with the DV defect is almost a factor of five smaller than that of the SW defect, especially for excitations that resonate with the first absorption band. This could provide a means to distinguish between the two defected CNTs, although this would be challenging as the differences are relatively small.

Overall our results also stand in very good agreement with the recent experiments of Kalbac and co-workers, where a low concentration of defects were created intentionally on part of an originally defect-free individual semi-conducting SWCNT. ${ }^{12}$ Doping was shown to lead to a broadening and to a decrease of the intensity maxima in the resonance profile. The small shift in the optical transitions due to doping that is observed in our study was also observed in the experiments where the maxima of the resonance profiles for the defective and pristine CNTs did not coincide; the spectra of the pristine CNT are excited by a $2.05 \mathrm{eV}$ laser excitation energy, while that of the defective one are excited by a $2.10 \mathrm{eV}$ energy pulse. In contrast to our findings, these experiments did not observe any new excitation bands in addition to the $S_{11}$ and $S_{22}$ absorption bands, however, this could be due to the small oscillator strengths of these induced excitations or their proximity to the main absorption bands as discussed before.

\section{Conclusions}

In summary, we have investigated for the pristine and defected $(7,0) \mathrm{CNT}$, the geometries, UV-vis absorption bands, and RR cross sections for the RBM and G-band using state-of-the-art DFT approaches. We show that the UV-vis spectrum, at least in the range of $0-7 \mathrm{eV}$ does not change significantly by the introduction of the defects. For example, the $S_{11}$ and $S_{22}$ excitation energies change by less than $0.1 \mathrm{eV}$ compared to the pristine CNT. We show that, on the other hand, defects introduced an order of magnitude reduction in the RR intensity for the RBM and G-band. These findings stand in agreement with recent experiments on CNTs where defects were introduced intentionally on a part of defect free SWCNT. In contrast to UV-vis spectra, or Raman shifts for that matter, the large difference in RR intensities reported for defected CNTs suggests that RR spectroscopy can provide a functionalization fingerprint to distinguish between pristine and defective CNTs. The G-band showed more sensitivity to the defects than the RBM, but in the low defect concentration limit it is not expected that this band would have substantial sensitivity based on the trends using two defect concentrations. The results are generally in agreement with our previous results on atom-decorated CNTs, which show that RR spectroscopy is an ultra-sensitive tool that can probe more general forms of the defect chemistry of CNTs.

\section{Acknowledgements}

W.A.S. is grateful for computing time provided by the University of Pittsburgh Center for Simulation and Modeling, Argonne Leadership Computing Facility at Argonne National Laboratory, which is supported by the Office of Science of the U.S. Department of Energy under contract DE-AC02-06CH11357, and the Extreme Science and Engineering Discovery Environment (XSEDE), which is supported by National Science Foundation grant number OCI-1053575. P.N. acknowledges financial support from the Swedish Research Council (Grant No. 621-2010-5014) and a grant for computing time at National Supercomputer Centre (NSC), Sweden.

\section{References}

1 S. Iijima and T. Ichihashi, Nature, 1993, 363, 603.

2 P. Singh, S. Campidelli, S. Giordani, D. Bonifazi, A. Bianco and M. Prato, Organic functionalisation and characterisation of single-walled carbon nanotubes, Chem. Soc. Rev., 2009, 38, 2214.

3 S. A. Hodge, M. K. Bayazit, K. S. Coleman and M. S. P. Shaffer, Unweaving the rainbow: a review of the relationship between single-walled carbon nanotube molecular structures and their chemical reactivity, Chem. Soc. Rev., 2012, 41, 4409.

4 X. Peng and S. S. Wong, Functional Covalent Chemistry of Carbon Nanotube Surfaces, Adv. Mater., 2009, 21, 625.

5 R. Saito, G. Dresselhaus and M. S. Dresselhaus, Physical Properties of Carbon Nanotubes, Imperial College Press, London, 1998.

6 A. Jorio, M. S. Dresselhaus and G. Dresselhaus, Carbon Nanotubes: Advanced Topics in the Synthesis, Structure, Properties and Applications, Springer, Berlin, 2008.

7 S. Picozzi, S. Santucci, L. Lozzi, L. Valentini and B. Delley, Ozone adsorption on carbon nanotubes: the role of StoneWales defects, J. Chem. Phys., 2004, 120, 7147-7152.

8 V. H. Crespi, M. L. Cohen and A. Rubio, In Situ Band Gap Engineering of Carbon Nanotubes, Phys. Rev. Lett., 1997, 79, 2093-2096.

9 J. A. Robinson, E. S. Snow, S. C. Baadescu, T. L. Reinecke and F. K. Perkins, Role of Defects in Single-Walled Carbon Nanotube Chemical Sensors, Nano Lett., 2006, 6, 1747-1751.

10 M. S. Dresselhaus, G. Dresselhaus, R. Saito and A. Jorio, Raman spectroscopy of carbon nanotubes, Phys. Rep., 2005, 409, 47.

11 F. Tuinstra and J. L. Koenig, Raman Spectrum of Graphite, J. Chem. Phys., 1970, 53, 1126-1130. 
12 M. Kalbac, Y.-P. Hsieh, H. Farhat, L. Kavan, M. Hofmann, J. Kong and M. S. Dresselhaus, Defects in Individual Semiconducting Single Wall Carbon Nanotubes: Raman Spectroscopic and In Situ Raman Spectroelectrochemical Study, Nano Lett., 2010, 10, 4619-4626.

13 J.-Y. Mevellec, C. Bergeret, J. Cousseau, J.-P. Buisson, C. P. Ewels and S. Lefrant, Tuning the Raman Resonance Behavior of Single-Walled Carbon Nanotubes via Covalent Functionalization, J. Am. Chem. Soc., 2011, 133, 16938.

14 V. N. Popov, L. Henrard and P. Lambin, Electron-phonon and electron-photon interactions and resonant Raman scattering from the radial-breathing mode of single-walled carbon nanotubes, Phys. Rev. B: Condens. Matter Mater. Phys., 2005, 72, 035436.

15 V. N. Popov and P. Lambin, Theoretical resonant Raman spectra of nanotube $(7,0)$ with point defects, Phys. Status Solidi, 2009, 246, 2602.

16 R. Saito, M. Fujita, G. Dresselhaus and M. S. Dresselhaus, Electronic structure of chiral graphene tubules, Appl. Phys. Lett., 1992, 60, 2204-2206.

17 C. D. Spataru, S. Ismail-Beigi, L. X. Benedict and S. G. Louie, Excitonic Effects and Optical Spectra of Single-Walled Carbon Nanotubes, Phys. Rev. Lett., 2004, 92, 077402.

18 H. J. Liu and C. T. Chan, Properties of $4 \AA$ carbon nanotubes from first-principles calculations, Phys. Rev. B: Condens. Matter Mater. Phys., 2002, 66, 115416.

19 D. A. Long, The Raman effect: a unified treatment of the theory of Raman scattering by molecules, Wiley, Chichester, 2002.

20 A. Mohammed, H. Ågren and P. Norman, Time-dependent density functional theory for resonant properties: resonance enhanced Raman scattering from the complex electric-dipole polarizability, Phys. Chem. Chem. Phys., 2009, 11, 4539.

21 W. A. Al-Saidi, S. A. Asher and P. Norman, Resonance Raman Spectra of TNT and RDX using Vibronic Theory, Excited-State Gradient and Complex Polarizability Approximations, J. Phys. Chem. A, 2012, 116, 7862.

22 P. Norman, D. M. Bishop, H. J. Aa. Jensen and J. Oddershede, Near-resonant absorption in the time-dependent self-consistent field and multi-configurational self-consistent field approximations, J. Chem. Phys., 2001, 115, 10323.

23 P. Norman, D. M. Bishop, H. J. Aa. Jensen and J. Oddershede, Nonlinear response theory with relaxation: the first hyperpolarizability, J. Chem. Phys., 2005, 123, 194103.

24 J. Kauczor, P. Jørgensen and P. Norman, On the efficiency of algorithms for solving Hartree-Fock and Kohn-Sham response equations, J. Chem. Theory Comput., 2011, 7, 1610.

25 S. Coriani, O. Christiansen, T. Fransson and P. Norman, Coupled-cluster response theory for near-edge X-ray absorption fine structure of atoms and molecules, Phys. Rev. A, 2012, 85, 022507.

26 S. Coriani, T. Fransson, O. Christiansen and P. Norman, An asymmetric Lanczos-chain driven implementation of electronic resonance convergent coupled cluster linear response theory, J. Chem. Theory Comput., 2012, 8, 1616.
27 D. Rocca, R. Gebauer, Y. Saad and S. Baroni, Turbo charging time-dependent density-functional theory with Lanczos chains, J. Chem. Phys., 2008, 128, 154105.

28 P. Seidler, M. B. Hansen, W. Györffy, D. Toffoli and O. Christiansen, Vibrational absorption spectra calculated from vibrational configuration interaction response theory using the Lanczos method, J. Chem. Phys., 2010, 132, 164105.

29 P. Giannozzi, et al., QUANTUM ESPRESSO: a modular and open-source software project for quantum simulations of materials, J. Phys.: Condens. Matter, 2009, 21, 395502.

30 J. P. Perdew, K. Burke and M. Ernzerhof, Generalized Gradient Approximation Made Simple, Phys. Rev. Lett., 1996, 77, 3865-3868.

31 A. Togo, F. Oba and I. Tanaka, First-principles calculations of the ferroelastic transition between rutile-type and $\mathrm{CaCl}_{2}$ type $\mathrm{SiO}_{2}$ at high pressures, Phys. Rev. B: Condens. Matter Mater. Phys., 2008, 78, 134106.

32 P. Norman, A perspective on nonresonant and resonant electronic response theory for time-dependent molecular properties, Phys. Chem. Chem. Phys., 2011, 13, 20519-20535.

33 W. A. Saidi and P. Norman, Probing Single-Walled Carbon Nanotube Defect Chemistry Using Resonance Raman Spectroscopy, Carbon, 2013, 67, 17, DOI: 10.1016/j.carbon.2013.09.045.

34 S. Berber and A. Oshiyama, Atomic and electronic structure of divacancies in carbon nanotubes, Phys. Rev. B: Condens. Matter Mater. Phys., 2008, 77, 165405.

35 X. Chang, J. Zhou, W. Fa and J. Dong, Vibrational properties and Raman spectra of single-wall carbon nanotubes with divacancy, J. Appl. Phys., 2010, 107, 103503.

36 H. Y. He and B. C. Pan, Characteristic vibrational modes of a single vacancy in a zigzag carbon nanotube, Phys. Rev. B: Condens. Matter Mater. Phys., 2008, 77, 073410.

$37 \mathrm{H}$. Ajiki and T. Ando, Aharonov-Bohm effect in carbon nanotubes, Phys. B, 1994, 201, 349-352.

38 C. Spataru, S. Ismail-Beigi, L. Benedict and S. Louie, Quasiparticle energies, excitonic effects and optical absorption spectra of small-diameter single-walled carbon nanotubes, Appl. Phys. A: Mater. Sci. Process., 2004, 78, 1129-1136, DOI: $10.1007 / \mathrm{s} 00339-003-2464-2$.

39 S. M. Bachilo, M. S. Strano, C. Kittrell, R. H. Hauge, R. E. Smalley and R. B. Weisman, Structure-Assigned Optical Spectra of Single-Walled Carbon Nanotubes, Science, 2002, 298, 2361-2366.

40 C. D. Spartaru, S. Ismail-Beigi, R. B. Capaz and S. G. Louie, in Carbon Nanotubes: Advanced Topics in the Synthesis, Structure, Properties and Applications, ed. A. Jorio, M. S. Dresselhaus and G. Dresselhaus, Springer, Berlin, 2008, p. 195.

41 S. Kilina, J. Ramirez and S. Tretiak, Brightening of the Lowest Exciton in Carbon Nanotubes via Chemical Functionalization, Nano Lett., 2012, 12, 2306-2312.

42 E. C. Girao, Y. Liebold-Ribeiro, J. A. Batista, E. B. Barros, S. B. Fagan, J. Mendes Filho, M. S. Dresselhaus and A. G. Souza Filho, Functionalization of single-wall carbon nanotubes through chloroform adsorption: theory and experiment, Phys. Chem. Chem. Phys., 2010, 12, 1518-1524. 